\title{
1 Parameter-free resolution estimation based on decorrelation analysis
}

A. Descloux ${ }^{1}$, K.S. Grußmayer ${ }^{1}$, A. Radenovic ${ }^{1}$

3

Affiliations

${ }^{1}$ École Polytechnique Fédérale de Lausanne, Laboratory of Nanoscale Biology, 1015 Lausanne,

5 Switzerland

6

$7 \quad$ Corresponding Authors

8 A. Descloux, email : adrien.descloux@epfl.ch, A. Radenovic, email : aleksandra.radenovic@epfl.ch 
11 Super-resolution microscopy opened diverse novel research directions by overcoming the classical

12 resolution limit. Revealing structures beyond the diffraction limit was made possible by exploiting the

13 fluorescent emission of individual fluorophores. Involving sample properties to apply these techniques

14 entails a redefinition of the resolution parameter. Here, we propose a new method for assessing the

15 resolution of individual super-resolved images based on image partial phase auto-correlation. The

16 novel algorithm is model-free and does not require any user-defined parameters. We demonstrate its

17 performance on a wide variety of imaging modalities, including diffraction-limited techniques. Finally,

18 we show how our method can be used to optimize image acquisition and post-processing in super-

19 resolution microscopy. 
Over the past decades, the field of microscopy was enriched with a broad range of novel imaging

23 methods, providing unprecedented insights into sub-cellular structures ${ }^{1,2}$. When designing a microscopy

24 experiment, one has to select an appropriate imaging modality taking into account the required spatial

25 and temporal resolution. The image quality greatly varies among different techniques and is influenced

26 by sample properties. Image formation for all microscopy techniques (coherent or incoherent,

27 diffraction-limited or super-resolution imaging) can be modelled as the convolution of a ground-truth

28 object with the specific point-spread function plus various method-related noise contributions. In Fourier

29 space, the object spectrum is multiplied by the transfer function of the system. The shape of the transfer

30 function depends on the imaging method employed, but common to all techniques is image low-pass

31 filtering, characterized by a cut-off frequency. This spatial frequency limit already known to $\mathrm{Abbe}^{3}$ is

32 generally expressed as $k_{c}=N A \frac{2 \pi}{\lambda}$, where $N A$ is the sine of the maximum collection angle multiplied by

33 the refractive index and $\lambda$ the illumination central wavelength, and corresponds to the resolution in

34 coherent imaging.

Super-resolution techniques overcome the diffraction limit by exploiting specific fluorophore properties such as stimulated emission or temporal fluctuations. Therefore, image resolution needs to be reconsidered by taking into account the fluorescent properties of the sample to establish a novel resolution measure for super-resolved imaging ${ }^{4}$. Ideally, this resolution criterion should work on a single image, be independent of the imaging method, have no user-dependent settings and be compatible with classical resolution. Such an estimator of resolution is of particular interest for autonomous adaptive

41 microscopes ${ }^{5,6}$ that require robust tools to automatically achieve and maintain optimal performance in 42 long-term imaging of biological samples. 
44 using two independent images of the same object for resolution estimation of electron microscopy images. The concept was quickly expanded to 3D via Fourier Shell Correlation by Harauz ${ }^{9}$ and several threshold concepts have been proposed $\left(0.5,0.143^{10}, 2 \sigma^{8,11}, \operatorname{SSNR}^{12}\right)$ to extract a resolution measure. Later, Banterle ${ }^{13}$ and Nieuwenhuizen ${ }^{14}$ independently extended, reconsidered and applied the method to assess the resolution of single-molecule localization microscopy (SMLM) images. Similar to electron microscopy, their method requires two stochastically-independent images of the same object and estimates the resolution by computing cross-correlations of Fourier space rings. The cut-off frequency is defined as the spatial frequency where the so-called FRC curve drops below a fixed value equal to 0.143 .

In SMLM, the two image realizations are typically accomplished by splitting the image series, i.e. acquiring two consecutive images under the same conditions. However, maintaining these conditions may be difficult due to, for example, bleaching or temporal fluctuations of the fluorescence signal. This is especially pertinent in live-cell imaging and significantly challenges the stationarity assumption of FRC. image without any further requirement or a priori knowledge. The algorithm expects only a nonsaturated, bandwidth-limited signal with adequate spatial sampling. This novel estimator is based on partial phase correlation and does not rely on any user-defined parameters. The algorithm is fast, uses only linear operations and enables the real-time objective assessment of image resolution and Signal to Noise Ratio (SNR). We successfully applied our new approach on a variety of microscopy data sets, ranging from widefield imaging to SMLM and STED microscopy. We show that our estimator can also be used to optimize image resolution, both during experiments and throughout data post-processing. 


\section{Decorrelation analysis}

To achieve objective threshold-free resolution estimation, we introduce a processing method termed decorrelation analysis. The main algorithm is divided into two steps. First, the Fourier transform of the image is computed after a standard edge apodization to suppress high-frequency artefacts. The

71 Fourier transform is normalized as $I_{n}(\boldsymbol{k})=\frac{I(\boldsymbol{k})}{|I(\boldsymbol{k})|}$. The input image $I(\boldsymbol{k})$ and its normalized version $I_{n}(\boldsymbol{k})$ are then cross-correlated in Fourier space using Pearson correlation and condensed in a single value between 0 and 1 (Fig. 1a). Second, the operation is repeated, but the normalized Fourier transform is additionally filtered by a binary circular mask of radius $r \in[0,1]$ expressed in normalized frequencies

(Fig. 1b). By repeating the calculation, we compute $d(r)$ which is expressed as

$$
d(r)=\frac{\iint \operatorname{Re}\left\{I(\boldsymbol{k}) I_{n}^{*}(\boldsymbol{k}) M(\boldsymbol{k} ; r)\right\} d k_{x} d k_{y}}{\sqrt{\iint|I(\boldsymbol{k})|^{2} d k_{x} d k_{y} \iint\left|I_{n}(\boldsymbol{k}) M(\boldsymbol{k} ; r)\right|^{2} d k_{x} d k_{y}}},
$$

where $\boldsymbol{k}=\left[k_{x}, k_{y}\right]$ denotes Fourier space coordinates, $I(\boldsymbol{k})$ the Fourier transform of the input image, $I_{n}(\boldsymbol{k})$ the normalized Fourier transform and $M(\boldsymbol{k} ; r)$ the binary mask of radius $r . \iint$ is the double integral over $k_{x}$ and $k_{y}$. For a detailed mathematical derivation and additional considerations, see Supplementary Material Section 1.

The core idea of the method is that by normalizing the Fourier transform of the input image, we balance the signal and noise contributions while the information of the object structure is preserved in the phase (the phase is responsible for organizing the constructive and destructive interferences of the complex exponentials to form the image, the amplitude plays only a minor role in this process). Taking a radius of the binary mask equal to 1 allows the extraction of the correlation value related to the original ratio of 
the white noise power spectrum is constant by definition (the normalization does not affect the signal).

87 If we add a bandwidth-limited signal to the image, the correlation value for $r=1$ will decrease (the $I(\boldsymbol{k}))$.
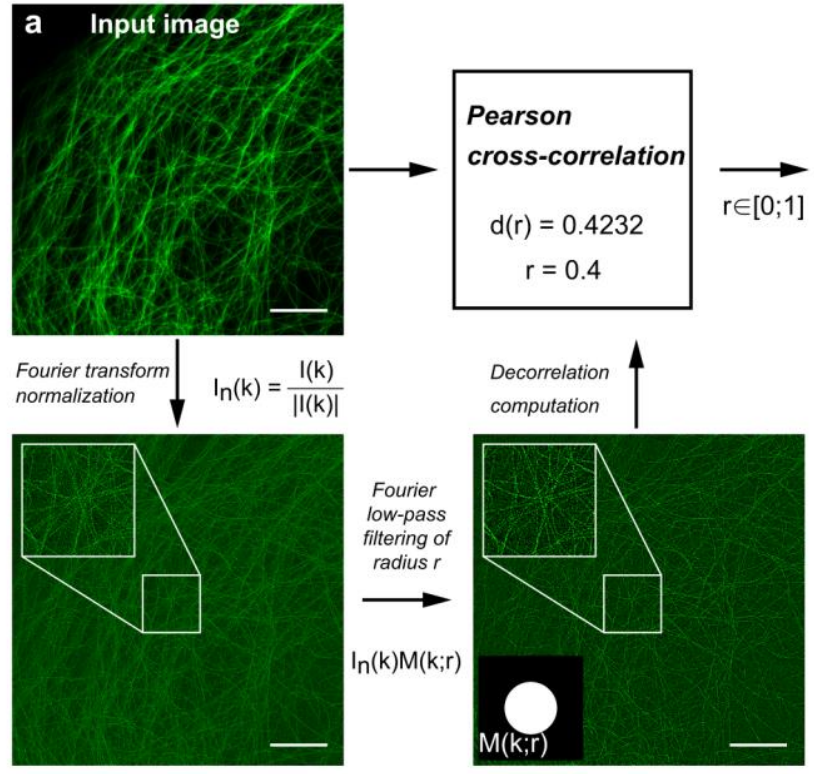

C

High-pass the input image with $N$ Gaussian filters

Curve computation $\Rightarrow$ Peak finding $\Rightarrow$ Resolution

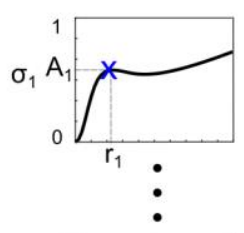

$\Rightarrow \quad\left[r_{1} ; A_{1}\right]$
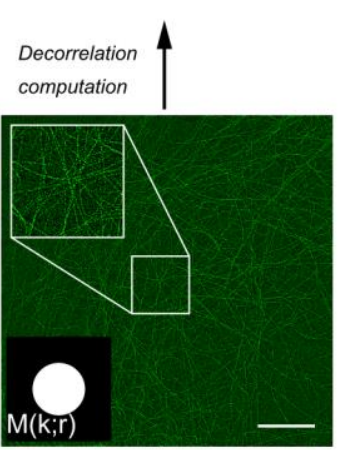

b

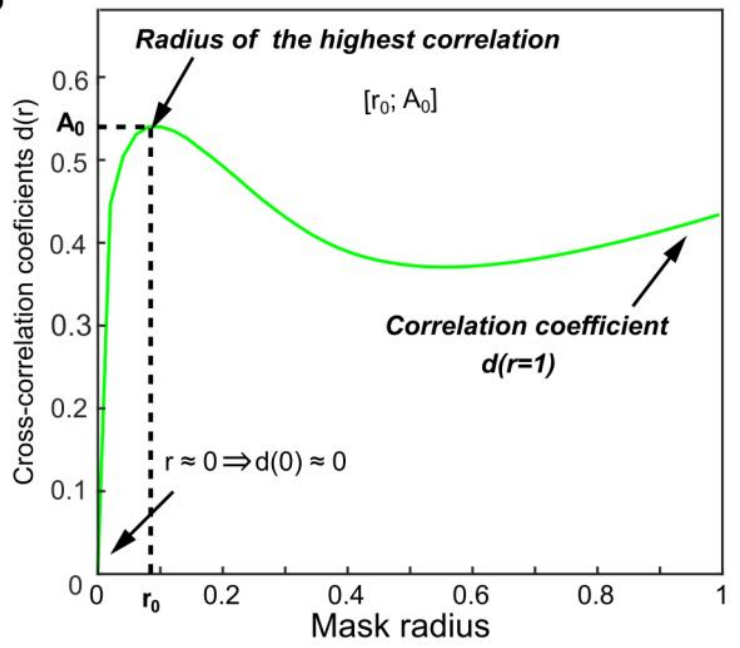

d
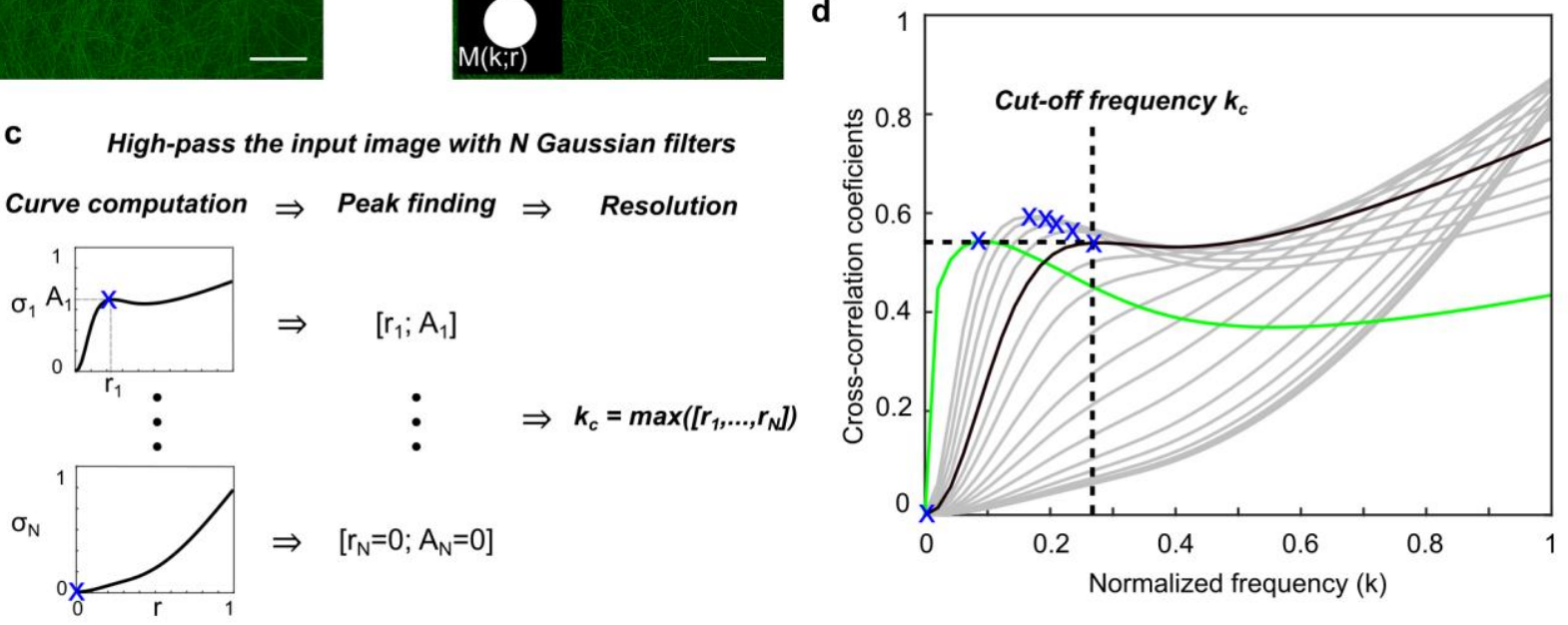

Figure 1: Image decorrelation analysis workflow. (a) Cross-correlation of the image with its Fourier-filtered normalized version. (b) Cross-correlation coefficient as a function of the mask radius. (c) High-pass filtering of the input image and resolution estimation. (d) The plot of all decorrelation functions computed for the image and resolution estimation; Green: Decorrelation function without any high-pass filtering, Grey: Decorrelation functions with high-pass filtering, Blue cross : Local maxima, Black: decorrelation function of highest frequency peak, Vertical dashed line: cut-off frequency $k_{c}$. Scale bar, $5 \mu \mathrm{m}$. 
correlation value will decrease linearly as a function of radius $r$. If we now add a signal, the decorrelation

99 function $d(r)$ will exhibit a local maximum of amplitude $A_{0}$ that indicates the spatial frequency $r_{0}$ of best noise rejection and signal preservation ratio. Restricting the mask further removes more signal than noise, therefore reducing the correlation below $A_{0}$ until it drops to 0 for $r=0$. The position $r_{0}$ of the

102 local maximum is therefore directly related to the spatial frequency distribution of the image and its amplitude $A_{0}$ is positively correlated with the image Signal-to-Noise Ratio (see Supplementary Fig. S1 for detailed plots of decorrelation function behaviour with respect to different noise statistics, aberrated transfer functions, various cut-off frequencies, various SNRs and high-pass filtering strength). For a detailed description of the algorithm, see Supplementary Material Section 1.1.

While being related to the spatial frequency content of the image, the position of the maximum does not directly indicate the resolution of the image. The input image is then subjected to a total of $N_{g}$ high-pass filters (spanning the range from weak to very strong filtering) in order to attenuate the energy of the low frequencies. For each filtered image, a decorrelation function is computed and the peak position $r_{i}$ and amplitude $A_{i}$ are extracted, generating a set of $\left[r_{i}, A_{i}\right]$ pairs (Fig. 1c). If the high-pass filtering removes too much signal, the decorrelation function will not exhibit a local maximum and the peak position and amplitude will both be set to 0 . We investigated two different strategies for the resolution estimation: selecting the highest frequency peak or selecting the peak corresponding to the highest geometric mean $\left(G M=\sqrt{r_{i} A_{i}}\right)$ (giving the same weight to the position and amplitude). After processing several simulations and microscopy images taken from different imaging modalities including

117 Confocal, STED, SIM, SOFI and PAINT, we observed that in all cases but confocal images, both criteria provide identical estimates of resolution (see Supplementary Material Section 1.2). We consequently define the resolution estimate as

$$
k_{c}=\max \left[r_{0}, \ldots, r_{N_{g}}\right]
$$


120 which corresponds to the local maximum of highest frequency (Fig. 1d). The resolution is then

121 resolution $=\frac{2 * \text { pixel size }}{k_{c}}$, where $k_{c}$ is expressed in normalized frequencies. By computing the resolution

122 with a varying sampling of $d(r)$ and a varying number of high-pass filtering $N_{g}$, we confirm the

123 robustness of the algorithm and estimate the precision of the algorithm to be about \pm 1 to $3 \mathrm{~nm}$,

124 independently of the type of image (see Supplementary Material Fig. S2). We confirmed, via simulations

125 of point emitters, MTFs, rings and crossing lines, that our resolution estimate depends linearly on the

126 frequency support of the image (see Supplementary Material Section 2.1, 2.2 and 2.3) and that the

127 amplitude of the local maximum $A_{0}$ before any filtering is directly correlated with the image SNR.

Instead of searching for the frequency at which the transfer function vanishes (which can only

129 be measured in the absence of noise), we estimate the highest frequency from the local maxima of the 130 decorrelation functions, enabling parameter-free image resolution estimation. The presented method

131 does not estimate the theoretical resolution as stated by Abbe, but rather the highest frequency with

132 high enough signal with respect to the noise. It provides a rapid and objective way to quantify the 133 frequency content of a single image without any user-defined parameter.

\section{Results}

To demonstrate the validity and broad applicability of the method, we processed nanorulers data provided by GATTAquant (courtesy of P. Tinnefeld and J. Schmied). DNA origami nanorulers emerged as

137 a platform for reliable performance evaluation across different super-resolution modalities. Their adaptability is due to the fact that one can design these self-assembled nanostructures by placing a defined number of fluorescent dye molecules in precise geometries ${ }^{16}$. The resolutions estimated by our algorithm are smaller than the mark-to-mark distances of the nanorulers, which corroborates the fact that they are resolved in all imaging modalities (see Supplementary Material Section 3). After establishing 
diffraction-limited (see Supplementary Material Section 4 for bright-field imaging) and super-resolution

144 microscopy images of biological structures, presented in the following. By imaging a z-stack of fluorescent

145 beads, we validated that our resolution estimate fits the expected resolution well. Our estimator

146 provides a unique tool to assess the alignment of an optical setup or the performance of a microscope

147 objective lens using a single experimental image (see Supplementary Material Section 5). All the

148 presented results have been processed using custom Matlab code (source code publicly available at

149 https://github.com/Ades91/ImDecorr.git). For ease of use, the algorithm has also been implemented in

150 Java and is available as an open source Image ${ }^{17}$ plugin (see Supplementary Material Section 6).

\section{Confocal/STED}

We started with confocal ${ }^{18}$ and Stimulated Emission Depletion (STED) microscopy ${ }^{19,20}$, both

point-scanning techniques that can be realized on the same setup thus allowing the transition from

diffraction-limited to super-resolution imaging. STED microscopy is a super-resolution method that uses

confocal illumination to excite the fluorophore and a donut-shaped depletion beam to de-excite (via stimulated emission) most of the surrounding fluorophores prior to fluorescence emission. The resolution of STED microscopy for a given fluorophore is dependent on the spatial and temporal coalignment of the two beams ${ }^{21}$, the shape, and the quality of the depletion beam and its power ${ }^{22}$. art imaging facilities, we imaged the microtubule network of COS-7 cells immuno-labelled with Abberior Star635P, both in confocal and STED mode (pulsed fluorescence excitation at $635 \mathrm{~nm}$ and pulsed STED depletion laser at $775 \mathrm{~nm}$ ). Fig. 2a-d show the resulting images and their corresponding decorrelation analysis. Throughout the manuscript, we used the following convention: the green line and the grey lines are the original decorrelation functions prior to high-pass filtering and post-high-pass filtering; the blue to black lines are the decorrelation functions with refined mask radius and high-pass filtering range; the 

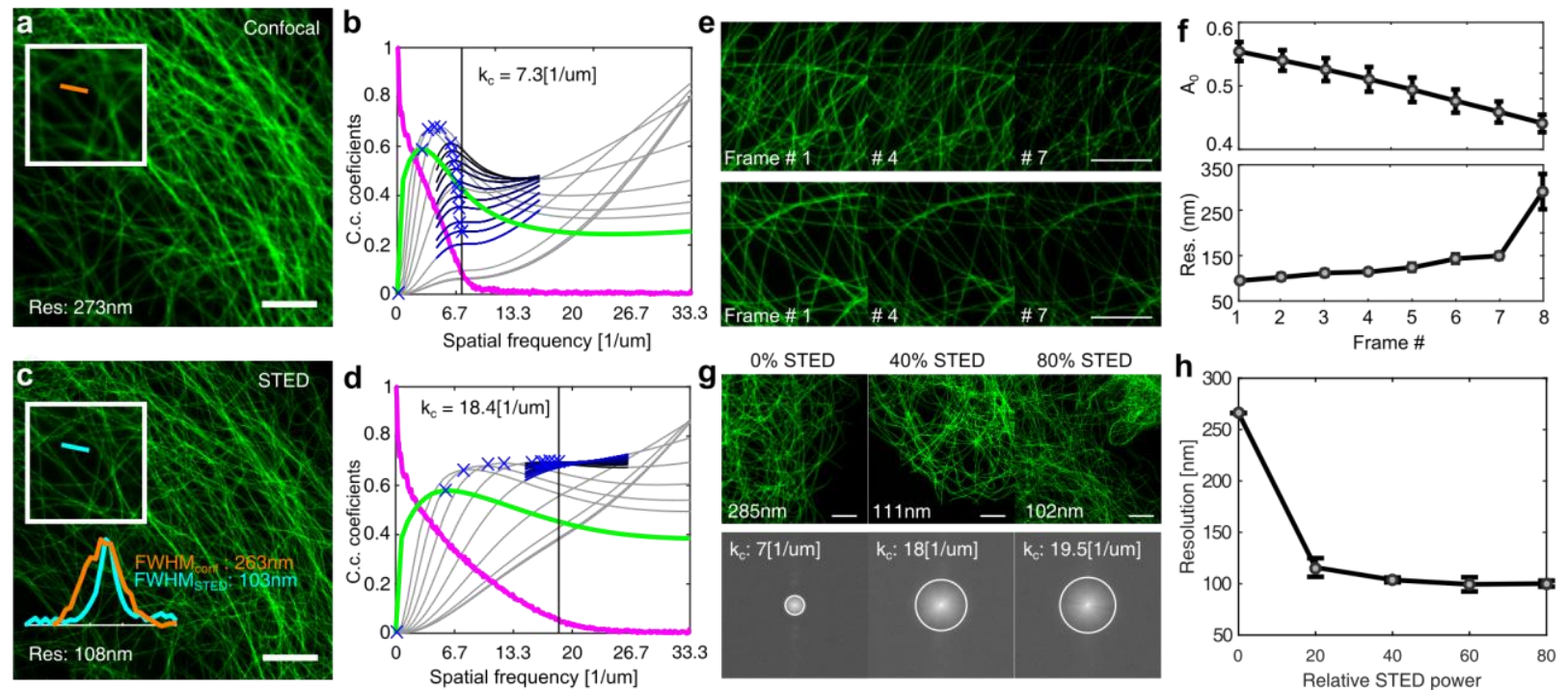

Figure 2: Confocal and STED. All images show microtubules in fixed COS-7 cells immunolabeled with Abberior Star 635P. (a) Confocal image and (b) its corresponding decorrelation analysis. Green line: decorrelation functions before high-pass filtering. Magenta line: Radial average of log of absolute value of Fourier transform of (a). Gray lines: all high-pass filtered decorrelation functions. Blue to Black lines: decorrelation functions with refined mask radius and high-pass filtering range. Blue crosses: all local maxima. Dashed vertical line : cut-off $k_{c}$ (for the sake of readability, we used the same color and style representation for all the subsequent analysis). (c) STED image of the same structure as in (a) with line profile of selected microtubule and (d) its corresponding decorrelation analysis. (e) Sequential STED imaging of two different cells (f) SNR estimator and resolution (average and standard deviation) of a total of 4 STED sequences as a function of time. (g) STED images as a function of STED power. The lower panel shows the corresponding Fourier space with indicated cut-off frequency. (h) Resolution as a function of STED power (average and standard deviation of 5 images per STED power). Scale bar, $5 \mu \mathrm{m}$. Image acquisition and sample details are provided in Table S1.

blue crosses indicate all the local maxima; the magenta line is the normalized radial-averaged log of the

absolute value of image Fourier transform. The image resolution is indicated as a black vertical line. As

resolution improvement over confocal imaging. We plotted in Fig. $2 c$ the manually selected line profile

of a microtubule cross-section, a method typically used to estimate the resolution. The measured

FWHMs are in good agreement with our estimates.

Fig. 2e and $f$ show the result of the analysis performed on a time series of eight consecutive STED

images (each STED image is the average of 8 scans; acquisition time per STED image of $15 \mathrm{sec}$ ) and a total

of four sequences (only two shown here). As expected, repeated imaging of the same structure gradually

bleaches the fluorophores, progressively degrading the SNR as indicated by the parameter $A_{0}$. We also 
close to the one observed in the confocal image. The bleaching is so strong that the structure is no longer

190 continuous. The proposed method confirms the expected degradation of image resolution and provides

a quantitative estimation of the image SNR and resolution. Fig. $2 \mathrm{~g}$ and $\mathrm{h}$ illustrate how our resolution estimation can be used to optimize STED imaging. Imaging under several STED illumination powers was performed, always adjusting the excitation power to maintain the optimal dynamic range of the image to avoid noise-limitation of STED resolution ${ }^{15}$ (for a total of 5 images per STED power, pixel size $40 \mathrm{~nm}$ ).

We see that using $20 \%$ of STED laser power has a significant improvement on the resolution (about 2.4 fold) but doubling the power only decreases the resolution by a factor of 1.1 as expected due to the nonlinear behaviour of STED. Further increases in STED power do not lead to significant changes in resolution, possibly due to a misalignment of the excitation and STED beam, imperfect "zero" of the STED doughnut as well as excess photo-bleaching and background induced by the STED beam ${ }^{20}$. We conducted additional experiments, investigating further acquisition parameters such as the STED delay, pixel size and comparing the performance of different dyes to choose the optimal label (see Supplementary increase the resolution (lifetime ${ }^{21}$, photostability ${ }^{23}$, spectra ${ }^{24}$, etc.). For pulsed STED experiments, the best resolution is reached when the depletion pulses immediately follow the excitation pulse. We used our resolution estimate as a readout to adjust the delay between the pulses in the Leica system. Figure S14c shows a drastic improvement in resolution at about $\Delta \mathrm{t}=1800 \mathrm{ps}$. Previously, an indirect strategy based on minimizing the remaining fluorescence intensity ${ }^{25}$ or an FRC resolution estimate was used ${ }^{15}$. objectively find the best acquisition settings and optimize sample preparation, including choice of dye, based on a single image of the sample of interest without imposing additional requirements on the data

211 acquisition scheme. In principle, it should as well be possible to use our resolution estimate to tune the 212 microscope alignment, e.g. to adjust the overlap of STED donut with confocal excitation spot. 
STED has also been used for high-resolution live-cell imaging, but care should be taken to avoid

215

sample damage due to high-depletion laser powers by special imaging procedures ${ }^{20}$. A super-resolution method that is widely used for imaging dynamics of living cells is Structured Illumination Microscopy ${ }^{26-28}$ (SIM). SIM aims at improving the lateral and axial resolution by multiple imaging of the sample with highfrequency illumination patterns ${ }^{29}$. The theoretical resolution improvement of SIM is linked to the frequency of the illumination. In practice, SIM resolution depends on the pattern modulation contrast, refractive index mismatch and local distortion of the pattern ${ }^{30}$. Fig. 3a shows the analysis of a pseudo widefield image, obtained by averaging the raw SIM sequence of actin filaments in U2OS cells ${ }^{31}$ labelled with Phalloidin-Atto488 (obtained on a Delta-Vision|OMX v4, courtesy of T. Huser). A resolution of about $253 \mathrm{~nm}$ is estimated by decorrelation analysis (cut-off of $7.9[1 / \mathrm{um}]$, the pixel size of $80 \mathrm{~nm}$; Fig. 3b). Fig. 3c shows the SIM reconstruction (see Supplementary Material Section 8 for the details of the reconstruction) of Fig. 3a and its corresponding decorrelation analysis (cut-off of $12.4[1 / \mathrm{um}]$, the pixel size of $45.8 \mathrm{~nm}$; Fig. 3d). We measure a resolution improvement of about 1.56. Measuring the position of the illumination peaks in the Fourier transform of the raw data (4.75 and $9.5[1 / \mathrm{um}]$ for the first and second diffraction order respectively), provides a way to estimate the theoretically expected resolution improvement. We observe that the contribution of the first diffraction order with the wide-field resolution $\left(\frac{7.9+4.75}{7.9} \approx 1.6\right)$ fits well with our estimation, indicating that the information encoded in the second diffraction order is not sufficiently contrasted. The use of more advanced reconstruction algorithms may improve this result.

So far, we only considered the global resolution, i.e. averaged over the whole image and in all directions. In order to account for non-isotropic resolution, we subdivide the Fourier space in sectors and compute the cut-off frequency as a function of the direction. This is referred to as sectorial resolution 
236 (see Supplementary Material Section 9). Fig. 3e shows a different pseudo widefield and SIM 237 reconstruction of the mitochondria network ${ }^{31}$ in U2OS cells (measured on a Zeiss Elyra S1, courtesy of 238 M. Sauer, resolution gain of 1.73). Fig.3f displays the Fourier transform of the SIM reconstruction, 239 overlapped with the sectorial resolution (solid white line) and the average resolution (dashed white
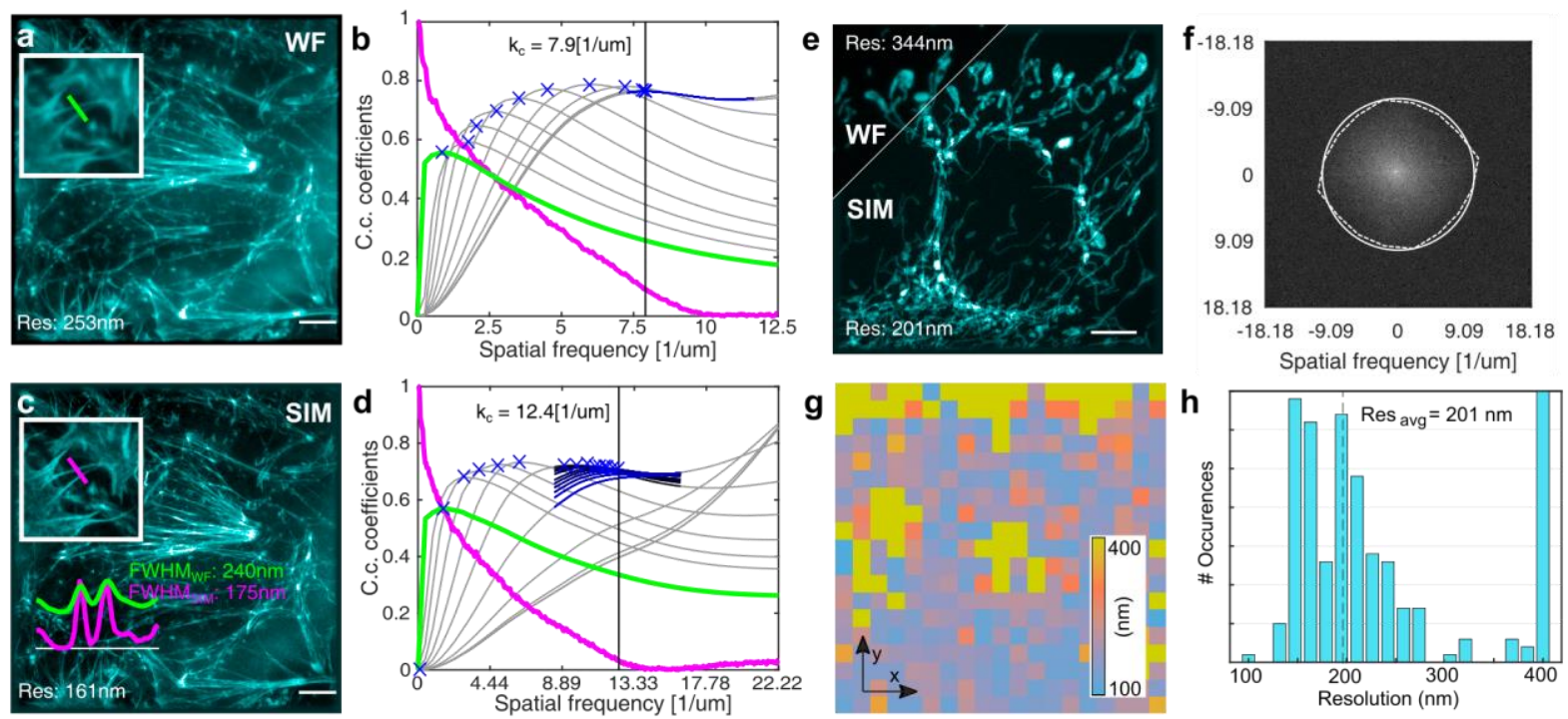

Figure 3: Widefield and SIM (a) Pseudo widefield image of the actin network in fixed U2OS cells labeled with phalloidin-Atto 488 (Courtesy of T. Huser). (b) Corresponding decorrelation analysis. (c) SIM reconstruction of (a) with selected cross-sections. (d) Corresponding decorrelation analysis. (e) Pseudo widefield and SIM reconstruction of mitochondria network in U2OS cells labeled with mitotracker (Courtesy of M. Sauer). (f) Sectorial resolution estimation (dashed white line) and average resolution (solid white circle). (g) Local resolution estimate of (e). (h) Histogram of local resolution shown in (g). Scale bar, 5 um. Image acquisition and sample details are provided in Table S1.

247 circle). Finally, by subdividing the image into smaller tiles (as was done for FRC $\left.\mathrm{in}^{14}\right)(70 \times 70$ pixels with an

overlap of 20 pixels), we can estimate the resolution over the whole field-of-view and reveal local variations in resolution (Fig. 3g). Due to the very weak signal in the top and center part of Fig.3e, corresponding sub-regions have very large resolution values. In order to preserve the dynamic range, all

histogram of resolutions (Fig. 3h), providing another perspective of the image. We also see that the average resolution is approximatively the median of all local resolutions. 

emitters. Stochastic Optical Fluctuation Imaging ${ }^{32,33}$ (SOFI) achieves super-resolution by computing high-

order spatiotemporal cumulants. SOFI processing is of interest to analyse because it predicts a resolution improvement of $1 / \sqrt{n}$, where $\mathrm{n}$ is the correlation order and $1 / n$ after deconvolution and brightness linearization. It provides an ideal test case for our resolution estimator.

Fig. 4a shows the results of SOFI analysis (up to 6th order) of MEF cells expressing paxillin labelled with mEos234 (courtesy of H. Deschout). Fig. 4b displays the results of the decorrelation analysis, where the raw cumulants follow the theoretical resolution improvement up to $70 \mathrm{~nm}$ for 6 th order linearized SOFI, indicating good blinking statistics. The deviation observed for the widefield (average of the whole sequence, here denoted as SOFI 1) and 2nd order SOFI can be attributed to sub-optimal out-of-focus light rejection. Similarly, the linearized SOFI cumulants, obtained by 10 iterations of Lucy-Ridcharson deconvolution and taking the nth root of the SOFI image, follow a similar trend close to the theoretical value. The deconvolution operation by itself constitutes an interesting case study for our algorithm. We show (see Supplementary Material Section 10) that the resolution can be set to any value, as a function of the input point-spread function and number of iterations of the deconvolution. It is the duty of the user to ensure that the resolution is not enhanced beyond the limit supported by the microscopy method. Our algorithm can thus be used to quantify deconvolution strength but not the validity of the deconvolution. subdividing the whole acquisition into sub-sequences, computing SOFI and averaging the results of the

275 sub-sequences ${ }^{35,36}$. By using our decorrelation analysis over the resulting SOFI image for various sub276 sequences lengths, we are able to identify the optimal resolution-SNR sub-sequence length for the given 277 input data. Fig. 4c shows the results of such an analysis (sub-sequence length ranging from 50 to 4000 

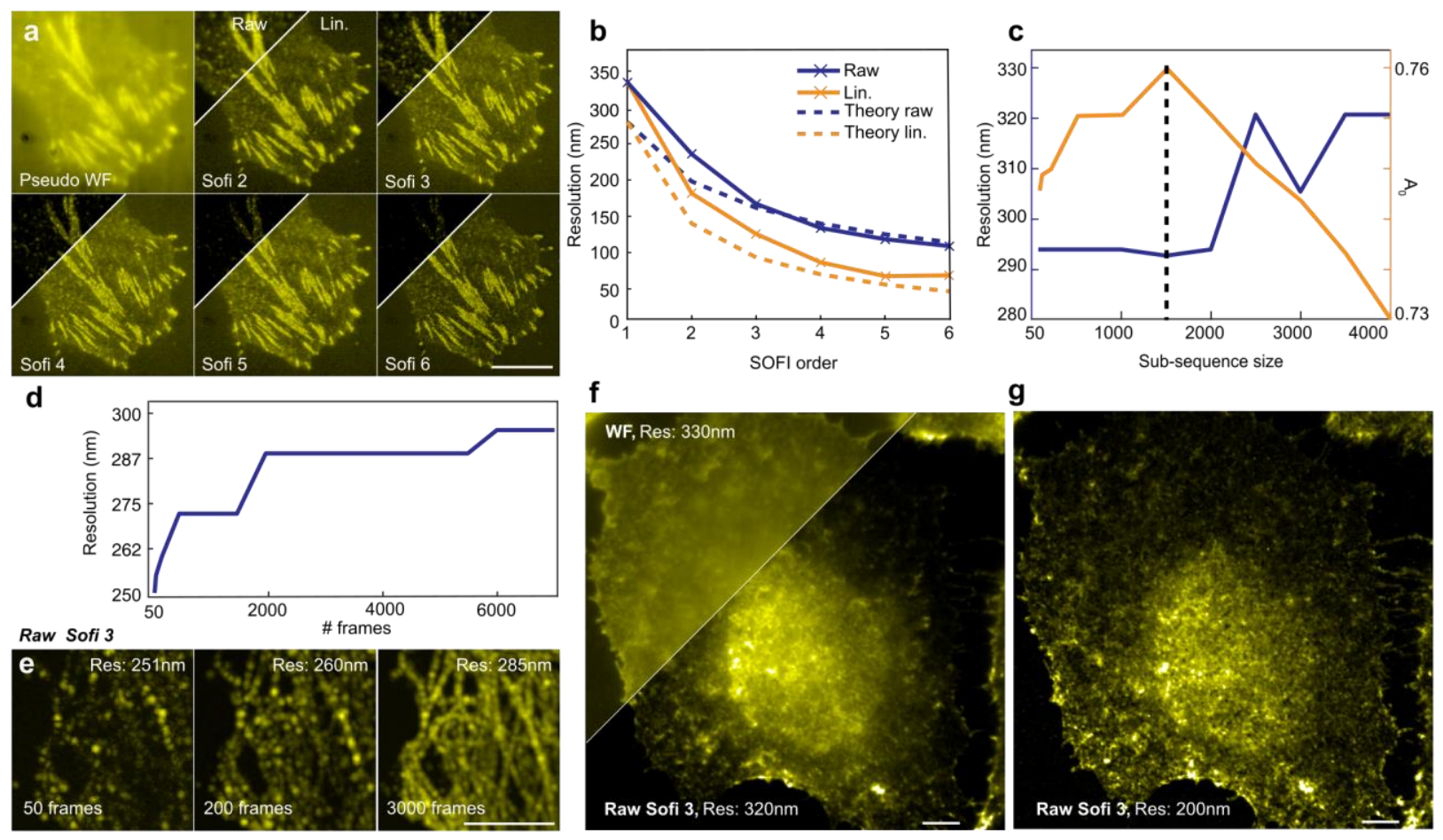

Figure 4: SOFI (a) Raw SOFI and Linearized SOFl images of focal adhesions in MEF cells expressing paxillin-mEos2 (courtesy of H. Deschout) shown up to order 6. (b) Estimated and theoretical resolution vs the SOFI order. (c-e) Cumulant analysis of microtubules in HeLa cells immunolabeled with Alexa Fluor 647. (c) Resolution and SNR estimate vs the sub-sequence size used for SOFI processing. (d) Resolution estimate vs number of frames. (e) Raw SOFI 3 images for 50, 200 and 3000 frames. (f) Un-optimized raw SOFI image of fixed HeLa cells labeled with wheat germ agglutinin-Atto 565 using "default" processing parameters. (g) Same images as (f) after optimization of the resolution. Scale bar, 5 Hm. Image acquisition and sample details are provided in Table S1.

286 frames), performed on a sequence of blinking Alexa647 targeting microtubules of HeLa cell (images optimal sub-sequence length of 1500 frames.

Fig. $4 \mathrm{~d}$ and $4 \mathrm{e}$ demonstrate how the total number of frames used to compute SOFI impacts the resolution in HeLa-cell microtubules labelled with Alexa 647. We see that 50 frames already allow the computation of a $3^{\text {rd }}$ order SOFI image. However, due to the blinking kinetics, many more frames are required to properly resolve the underlying structure. We also see that the resolution is slightly better when the image consists only of sparse point-like structures. This is due to the fact that our algorithm estimates the resolution from a single image. Using only 50 frames, the algorithm sees a sparse distribution of high-frequency dots. As we include more frames in the analysis, a larger-scale structure 
containing more low frequencies starts to emerge, leading to a slightly modified resolution estimate.

297 Finally, Fig. $4 \mathrm{f}$ shows a raw $3^{\text {rd }}$ order SOFI image of HeLa cells stained with wheat germ agglutinin-Alexa

298 488, processed with default parameters (first 1000 frames removed and sub-sequence length of 500

299 frames). Fig. 4g shows the same image, after optimization of the sub-sequence length and the number

300 of frames to be removed at the beginning and the end of the acquired data to obtain the best resolution

301 (1800 first frames removed, subsequence length of 1000, no frames removed at the end of the

302 sequence). The optimized processing procedure results in a 1.6-fold improvement of resolution

303 compared to the starting image.

\section{Localization microscopy}

305 Finally, we applied our method on Single Molecule Localization Microscopy ${ }^{37-39}$ (SMLM) data. In this case,

306 super-resolution is achieved by the individual localization of a subset of sparsely and stochastically

307 blinking emitters in successive image frames. By fitting the emission point-spread functions, single- and

308 multiple-emitter positions can be determined with nanometric accuracy ${ }^{40,41}$. To estimate the resolution,

309 our method requires a rendered image. This is typically realized by replacing a filtered set of localization

310 events by a 2D Gaussian of standard deviation equal to the localization uncertainty. The filtering step

311 consists of rejecting unphysical or poor localization events. We validated, using simulations, that our

312 algorithm is also able to correctly estimate the resolution of a localization-based image (see

313 Supplementary Material, Section 11 and Fig. S17). Fig. 5a shows a STORM image of immuno-labelled

314 microtubules (Abberior Flip 565, 16000 frames, localized using single-emitter least square fitting in

315 ThunderSTORM ${ }^{42}$ with default parameters). Each localization was rendered (in Matlab, the pixel size of

$31610 \mathrm{~nm}$ ) as a normalized Gaussian with standard deviation equal to the localization uncertainty. 


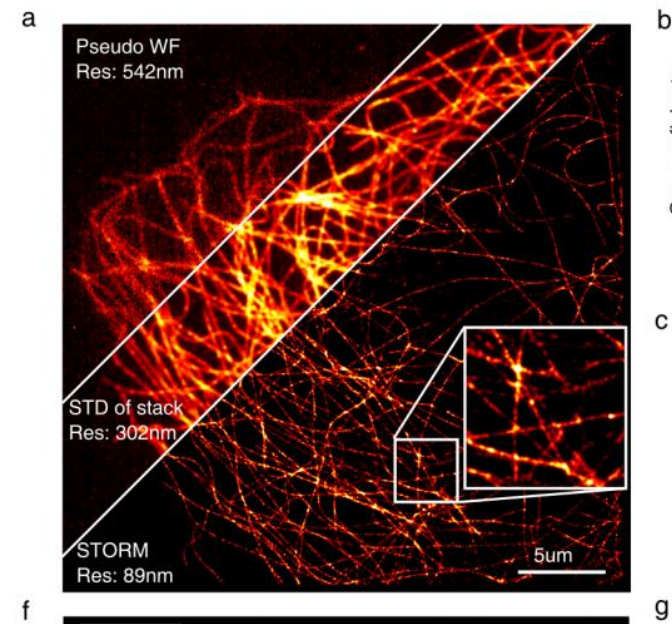

f

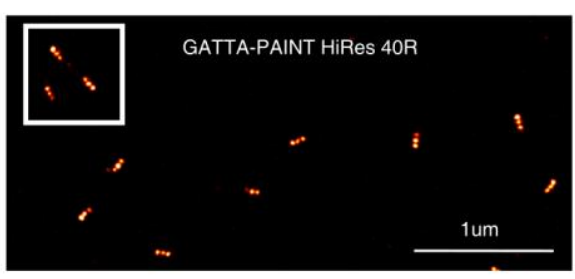

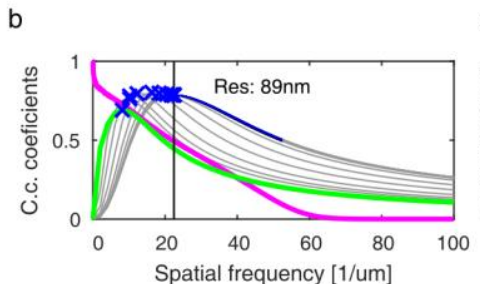

C

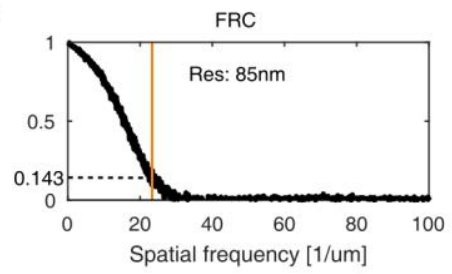

g

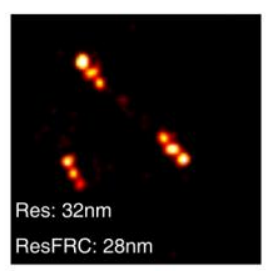

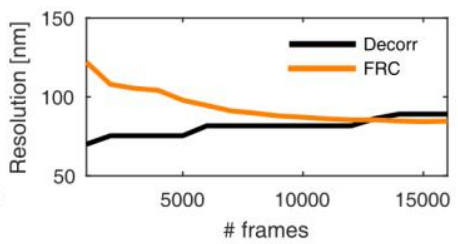

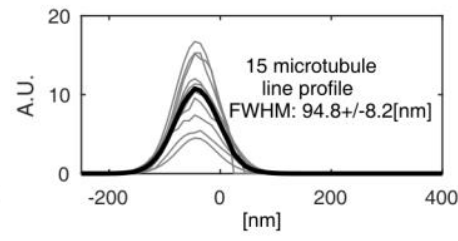

h

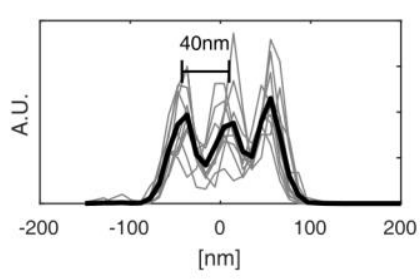

Figure 5: Localization microscopy (a) Pseudo widefield, standard deviation and STORM image of microtubules in COS 7 cells labelled with Abberior Flip 565. (b) Decorrelation analysis of (a). (c) Fourier Ring Correlation analysis of (a). (d) Decorrelation resolution (black line) and FRC resolution (orange line) as a function of a number of frames. (e) Line profile of 15 randomly selected microtubule cross-sections ( $f$ ) ( $f$ ) GATTAquant PAINT image of HiRes 4OR nanoruler with mark-to-mark distances of $40 \mathrm{~nm}$. (g) Zoom of (f) and decorrelation and FRC resolution estimate. (h) 10 line profiles of HiRes 4OR molecules indicating a resolution better than $40 \mathrm{~nm}$. Image acquisition and sample details are provided in Table S1.

With self-blinking dyes ${ }^{43,44}$, it is not possible to take a widefield image. We thus obtained a pseudo widefield (WF) and standard deviation (STD) image by computing the temporal average and standard deviation of all the frames. We estimate a resolution of $542 \mathrm{~nm}$ for the pseudo widefield, due to the low SNR of the image and a resolution of $302 \mathrm{~nm}$ for the STD image. Finally, we estimate a resolution of $89 \mathrm{~nm}$ for the STORM image. Fig. 5b and c display the corresponding decorrelation resolution estimate and FRC curve, respectively (obtained by splitting the localizations in two odd and even localization subsets to generate two independent realizations). Both estimates agree on the resolution, with FRC (estimated resolution of $85 \mathrm{~nm}$ ) being slightly more optimistic, which is consistent with its behaviour observed in simulations and reported recently by Marsh et $\mathrm{al}^{40}$.

Fig. 5d shows how our method and FRC resolution vary as a function of the number of frames.

We observe a drastic difference in the predicted resolution between the two methods only up to 5000 
336 frames. This is due to the fact that FRC requires two images instead of one. For a low number of frames,

337 the localization events are too sparse to produce significant correlations, leading to a large resolution

338 estimate. On the other hand, our method only considers a single image, which is constituted of sparse

339 Gaussians with no apparent structure but a very good SNR (since there is no noise in a rendered

340 localization image). This leads to a very optimistic resolution estimate with the localization uncertainty

341 as a lower bound. As we increase the number of frames, the two random subsets of localization events

342 start to correlate and the FRC resolution estimate starts to decrease. Similarly, as we include more

343 localization events, a larger-scale structure (hence containing low spatial frequencies) starts to emerge.

344 Consequently, our resolution estimate increases as the structure is built up. We finally observe that both

345 methods converge at approximately the same speed at around 12000 frames, with FRC estimating a

346 slightly better resolution ${ }^{45}$. Fig 5 e shows a total of 15 microtubules cross-sections randomly selected over

347 Fig. 5a. The apparent average microtubule diameter is about $95 \mathrm{~nm}$, which is consistent with the

348 resolution estimate and the secondary immunostaining, which increases the apparent microtubule diameter by $10-30 \mathrm{~nm}^{46,47}$.

Fig. $5 f$ shows a rendered image of GATTAquant HiRes 40R nanorulers ${ }^{48}$ (courtesy of P. Tinnefeld

and J. Schmied). Fig. $5 \mathrm{~g}$ shows a closeup image of three molecules showing that the three point-source spaced by $40 \mathrm{~nm}$ can be resolved. Our algorithm estimates a resolution of $32 \mathrm{~nm}$ while FRC estimates a resolution of $28 \mathrm{~nm}$, again being slightly more optimistic than our method. Fig. 5h shows a total of 10 line profiles of individual molecules (grey lines) and their average (solid black line).

Finally, it has been shown that multiple blinking events can severely impact the FRC resolution estimate by introducing spurious correlations ${ }^{14}$. While this effect can in principle be mitigated, it requires 357 an accurate estimation of the underlying blinking statistics ${ }^{49}$. We show (see Supplementary Material Fig. 358 S18) that our resolution estimate is independent on the probability of multiple blinking event, as our method does not require any assumption on the blinking kinetics. 
We proposed a new method for parameter-free resolution and SNR estimation of a single microscopy image. We were able to circumvent the need for a threshold by introducing a new form of partial phase correlation. In principle, our method can be applied to any imaging technique, including electron microscopy, atomic force microscopy, X-ray tomography and live-cell imaging. Here, we demonstrated its broad applicability by applying it to various types of microscopy images, ranging from bright field to single-molecule localization microscopy images. By processing nanoruler data, we showed that the method can be used to quantitatively assess the resolution which enabled an optimization of imaging parameters, post-processing and reconstruction of acquired data. Our approach provides a new and objective way to quantify the effective resolution in super-resolution microscopy. The extension of our method to 3D would require a reformulation of our algorithm in the spherical coordinate. processing specialist. We provide a unique and novel approach for resolution estimation based on the analysis of a single image that can be used for image processing optimization and image reconstruction microscopy setup characterization and optimization as well as for automated microscopes. We hope that

376 our method will be adopted by the ever-growing microscopy community as an everyday tool, helping 377 them to achieve high-quality research.

\section{Materials and methods}

\section{Cell culture}

$380 \mathrm{HeLa}$ and COS-7 cells were cultured at $37{ }^{\circ} \mathrm{C}$ and $5 \% \mathrm{CO}_{2}$ using DMEM high glucose with pyruvate $(4.5 \mathrm{~g}$

$381 \mathrm{I}^{-1}$ glucose, with GlutaMAX ${ }^{\top \mathrm{M}}$ supplement) supplemented with $10 \%$ fetal bovine serum and $1 \mathrm{x}$ penicillin382 streptomycin (all gibco ${ }^{\circ}$, Thermo Fisher Scientific). 
384 Cells were seeded in Lab-tek ${ }^{\bullet}$ II chambered cover slides (nunc) or on $18 \mathrm{~mm}$ high-precision No. 1.5 385 borosilicate coverslips (Marienfeld) in 12 well plates (Thermo Fisher Scientific) 1-2 days before fixation 386 in DMEM (see cell culture) or DMEM high glucose w/o phenol red (4.5 $\mathrm{g} \mathrm{l}^{-1}$ glucose) supplemented with $3874 \mathrm{mM} \mathrm{L-gluthamine,} 10 \%$ fetal bovine serum and 1x penicillin-streptomycin (all gibco ${ }^{\circ}$, Thermo Fisher 388 Scientific).

\section{HeLa cells:}

Cells were washed twice in pre-warmed buffer (microtubule stabilizing buffer (MTSB): 100 mM PIPES pH

6.8, $2 \mathrm{mM} \mathrm{MgCl}$, $5 \mathrm{mM} \mathrm{EGTA}$ or PBS for wheat germ agglutinin (WGA) staining), followed by application of pre-warmed fixation buffer (3.7\% paraformaldehyde (PFA), $0.2 \%$ Triton X-100 in MTSB or $3.7 \%$ paraformaldehyde (PFA) in PBS for wheat germ agglutinin (WGA) staining) for 15 min at room temperature (RT). Cells were then washed three times for 5 min each with $1 \times$ PBS and stored in $50 \%$ glycerol in $1 \times$ PBS at $4{ }^{\circ} \mathrm{C}$ or the immunostaining protocol was continued to prepare samples for fluorescence imaging. Fixed and permeabilized cells were blocked with $3 \%$ BSA in 1 x PBS and $0.05 \%$ Triton X-100 for 60 min at RT or overnight at $4{ }^{\circ} \mathrm{C}$.

398 Cells fixed without permeabilization were stained with $5 \mathrm{ng} \mathrm{m}^{-1}$ WGA-Atto 565 for $10 \mathrm{~min}$ followed by three times 5 min washes with $1 \times$ PBS. The blocked samples with prior permeabilization were 400 immediately incubated with a mix of primary anti-tubulin antibody (1 $\mathrm{mg} \mathrm{ml}^{-1} \mathrm{DM} 1$ a mouse monoclonal 401 (ab80779) 1:150 dilution, Abcam) in antibody incubation buffer for 60 min at RT (AIB: 1 \% BSA in 1 x PBS and $0.05 \%$ Triton X-100). Cells were then washed three times for 5 min each with AIB, followed by 403 incubation with donkey anti-mouse-Alexa Fluor 647 antibody (0.005 $\mathrm{mg} \mathrm{ml}^{-1}$ Invitrogen) for $60 \mathrm{~min}$ at RT. 404 This and all subsequent steps were performed in the dark. All cells were again washed three times for 5 
min each with AIB and incubated for 15 min post-fixation with $2 \%$ PFA in 1 x PBS followed by three 5 min washes with PBS. Cells were imaged immediately or stored in $50 \%$ glycerol in 1 x PBS at $4{ }^{\circ} \mathrm{C}$ until imaging.

\section{cos-7 cells:}

408 The protocol is similar as described previously by Chazeau et al. ${ }^{50}$. Cells were washed twice in prewarmed DMEM w/o phenol red (see cell culture) following 90s incubation with extraction buffer 410 (microtubule stabilizing buffer 2 (MTSB2: 80 mM PIPES, 7 mM MgCl2, 1 mM EGTA, 150mM NaCl, 5mM

411 D-glucose adjust $\mathrm{pH}$ to 6.8 using $\mathrm{KOH}$ ) with freshly added $0.3 \%$ Triton X-100 (AppliChem) and $0.25 \%$ 412 glutaraldehyde (stock solution 50\% electron microscopy grade, Electron Microscopy Sciences). 413 Immediately afterwards, pre-warmed $4 \%$ paraformaldehyde (PFA) in PBS was incubated for 15 min at 414 room temperature (RT). Cells were then washed three times for 5 min each with $1 \times$ PBS and stored in $41550 \%$ glycerol in $1 \times$ PBS at $4{ }^{\circ} \mathrm{C}$ or the immunostaining protocol was continued. Next, a freshly prepared 416 solution of $10 \mathrm{mM} \mathrm{NaBH} 4$ in 1x PBS was incubated on the cells for 7 minutes followed by one quick wash 417 in 1xPBS, and two washes 10min 1xPBS on an orbital shaker. Cells were permeabilized in PBS with 0.25 $418 \%$ Triton X-100 for 7min followed by blocking with blocking buffer (BB: $2 \%(w / v)$ BSA, 10mM glycine, $41950 \mathrm{mM}$ ammonium chloride $\mathrm{NH}_{4} \mathrm{Cl}$ in PBS pH 7.4 for 60 min at RT or overnight at $4{ }^{\circ} \mathrm{C}$.

420 The blocked samples were immediately incubated with primary anti-tubulin antibody (clone B-5-1-2 ascites fluid 1:100-1:200 dilution, Sigma-Aldrich) in BB for 60 min at RT. Cells were then washed three

422 times for 5 min each with BB, followed by incubation with either donkey anti-mouse-Alexa Fluor 647 423 antibody for SOFI imaging (donkey anti-mouse $(\mathrm{H}+\mathrm{L})$ highly cross-adsorbed at $0.005 \mathrm{mg} \mathrm{ml}^{-1}$ Invitrogen), 424 donkey anti-mouse-AbberiorFlip565 for SMLM imaging (preparation see below at 1:200 dilution) or goat 425 anti-mouse-AbberiorStar635P (at 0.005-0.01 $\mathrm{mg} \mathrm{m}^{-1}$ Abberior), goat anti-mouse-Atto594 (at 0.0025$4260.005 \mathrm{mg} \mathrm{ml}^{-1}$ Atto-tec), donkey anti-mouse-Alexa Fluor 594 antibody (donkey anti-mouse (H+L) highly 427 cross-adsorbed at $0.005 \mathrm{mg} \mathrm{ml}^{-1}$ Invitrogen)or donkey anti-mouse-biotin (Biotin-SP (long spacer) 
428 AffiniPure Donkey Anti-Mouse IgG $(\mathrm{H}+\mathrm{L})$, at 1:200 Jackson ImmunoResearch) for STED imaging for 60 min

429 at RT. This and all subsequent steps were performed in the dark. All cells were again washed three times 430 for 5 min each with AIB and incubated for 15 min post-fixation with $2 \%$ PFA in $1 \times$ PBS followed by three

4315 min washes with PBS. Cells with biotinylated secondary antibody were additionally incubated with 432 streptavidin-Atto490LS (at $0.01 \mathrm{mg} \mathrm{ml}^{-1}$ Atto-tec) in PBS for 30min followed by 3 washes for 5 min in PBS 433 before post-fixation. Cells were imaged immediately or stored in $50 \%$ glycerol in $1 \times$ PBS at $4{ }^{\circ} \mathrm{C}$ until 434 SOFI or SMLM imaging. For STED microscopy, cells were mounted on a coverglass slide (Thermo Fisher 435 Scientific) using Mowiol-DABCO (preparation see below) and allowed to harden for at least $24 \mathrm{~h}$ at RT. 436 Cells were imaged within 1 week of sample preparation.

\section{Preparation of labeled proteins}

$4382 \mathrm{mg} \mathrm{ml}^{-1}$ donkey anti-mouse $(\mathrm{H}+\mathrm{L})$ highly cross-adsorbed antibody (Invitrogen) was incubated with 439 AbberiorFlip565-NHS (Abberior) and $2 \mathrm{mg} \mathrm{ml}^{-1}$ WGA (Vector Labs) was incubated with Atto565-NHS 440 esther (Atto-tec) at a ratio of 1: 6 for $1 \mathrm{~h}$ at RT while shaking with the $\mathrm{pH}$ raised to 8.3 using sodium 441 bicarbonate. The mixture was purified using illustra NAP Columns (GE Healthcare) according to 442 manufacturer's instructions and eluted with slightly acidic PBS to recover the labeled antibody at neutral $443 \mathrm{pH}$. The protein concentration was estimated by absorption spectrometry to $<1.5 \mathrm{mg} \mathrm{ml}^{-1}$ donkey anti444 mouse AbberiorFlip565 and $0.5 \mathrm{mg} \mathrm{ml}^{-1}$ WGA-Atto565.

\section{Imaging buffer and embedding medium}

446 The samples for SOFI using Alexa Fluor dyes were imagined in a $50 \mathrm{mM}$ Tris- $\mathrm{Hcl} \mathrm{pH} 8.0,10 \mathrm{mM} \mathrm{NaCl}$ 447 buffer containing an enzymatic oxygen scavenging system ( $2.5 \mathrm{mM}$ protocatechuic acid (PCA) and $50 \mathrm{nM}$ 448 Protocatechuate- 3,4-Dioxygenase from Pseudomonas Sp. (PCD) with >3 Units $\mathrm{m} \mathrm{g}^{-1}$ ) and a thiol (2449 Mercaptoethylamine). The thiol and a stock solution of $100 \mathrm{mM} \mathrm{PCA}$ in water, pH adjusted to 9.0 with $450 \mathrm{NaOH}$, were always prepared fresh. PCD was aliquoted at a concentration of $10 \mu \mathrm{M}$ in storage buffer 
451 (100 mM Tris- $\mathrm{HCl} \mathrm{pH} \mathrm{8.0,50 \%} \mathrm{glycerol,} 50 \mathrm{mM} \mathrm{KCl}, 1 \mathrm{mM}$ EDTA) at $-20^{\circ} \mathrm{C}$ and thawn immediately before

452 use. The samples for SMLM using Abberior Flip 565 were imaged in 1x PBS. Mowiol-DABCO for STED 453 embedding was prepared as described in the manufacturer protocol (Roth Gebrauchsanweisung Mowiol 454 488). Aliquots were kept at -20C and thawn immediately before use.

\section{Microscope setups}

\section{Widefield, SOFI and SMLM}

457 Data for Fig. 4e-g, Fig. 5, Fig. S3a-d and Fig. S7a were acquired on a standard widefield custom build 458 microscope. A total of 4 illumination laser lines (405nm 200 mW Roithner; 488nm 200mW Toptica; $459561 \mathrm{~nm} 350 \mathrm{~mW}$ Quantum laser; 635nm 1W Roithner) are collimated, expanded and combined with 460 dichroic filters. The beams are then cropped with a rectangular aperture of approx. $7.2 \mathrm{~mm}$ placed in the 461 conjugated object plane, resulting in a $120 \times 120 \mu \mathrm{m}$ field of view. The beams are then focused with an 462 achromatic lens $(f=200 \mathrm{~mm})$ and reflected by a $3 \mathrm{~mm}$ thick Quad Line Beamsplitter (R405/488/561/635; 463 Semrock) in the back focal plane of the objective (Nikon 60x/1.27NA SR water immersion). The 464 fluorescence signal is focused on the camera (Orca Flash 4.0; Hamamatsu) with a 200mm achromatic 465 lens. The sample position is controlled in X and $Y$ by a Scan-plus IM 120x80 (Marzheuser) and in Z by a 466 Nano-Z piezo nanopositioner (Mad City Labs). All acquisitions were performed using Micromanager. The 467 laser intensities used in the experiments can be found in Table S1.

\section{Confocal and STED}

Confocal and STED microscopy was performed at the EPFL bioimaging and optics platform (BIOP) using a

470 Leica SP8 STED 3X. The setup consists in a Leica DMi 8 inverted microscope body equipped with a white 471 light laser (470-670nm) and a Leica HC PL APO 100X/1.40 oil objective for STED. For STED imaging we 472 used the $775 \mathrm{~nm}$ pulsed depletion laser and detected the fluorescence on HyD detectors. $100 \% 775 \mathrm{~nm}$ 473 laser power corresponds to $403 \mathrm{~mW}, 100 \% 633 \mathrm{~nm}$ laser power corresponds to $1.12 \mathrm{~mW}, 100 \% 488 \mathrm{~nm}$ 
474 laser power corresponds to $0.33 \mathrm{~mW}$ and $100 \% 520 \mathrm{~nm}$ laser power corresponds to $0.43 \mathrm{~mW}$ (power

475 measurements were performed after the objective by the BIOP). The laser powers and other acquisition

476 parameters used in the experiments can be found in Table S1.

\section{Data processing}

478 The algorithm is implemented in MATLAB (Mathworks) and ImageJ. All the codes are available upon 479 request.

\section{Acknowledgments}

481 The authors would like to thank Dr. T. Lukes and Prof. T. Laser for insightful discussions. We also thank 482 Dr. H. Deschout, Dr. M. Muller, Prof. T. Huser, Prof. M. Sauer for sharing SOFI and SIM data. We also 483 thank Dr. J. Schmied and Prof. P. Tinnefeld for sharing GATTAquant nanoruler data. This project has been 484 partly funded by the European Union's Horizon 2020 research and innovation program via grant 485 686271/SEFRI 16.0047. K.G. acknowledges the support from the Horizon 2020 Framework Program of 486 the European Union under the Marie Skłodowska-Curie Grant Agreement No. [750528]. A.D. and A.R. 487 acknowledge the support from Zeiss IDEAS center. We would like to thank EPFL Biolmaging \& Optics Core 488 Facility (EPFL-BIOP) for access to confocal and STED microscopes.

\section{Author contributions}

490 A.D. proposed and developed the method, processed all the presented data, wrote the Matlab and Java 491 code. K.S.G. prepared all the cells and performed measurements. A.R. supervised the research. A.D. 492 wrote the manuscript with comments of all co-authors at all stages.

\section{Data and materials availability}

494 All data needed to evaluate the conclusions in the paper are present in the paper and/or the 495 Supplementary Materials. Additional data related to this paper may be requested from the authors. 
497 1. Sahl, S. J., Hell, S. W. \& Jakobs, S. Fluorescence nanoscopy in cell biology. Nat. Rev. Mol. Cell Biol. $498 \quad 18,685-701(2017)$.

499 2. Sigal, Y. M., Zhou, R. \& Zhuang, X. Visualizing and discovering cellular structures with super500 resolution microscopy. Science 361, 880-887 (2018).

501 3. Abbe, E. Beiträge zur Theorie des Mikroskops und der mikroskopischen Wahrnehmung. Arch. für 502 Mikroskopische Anat. 9, 413-418 (1873).

503 4. Sheppard, C. J. R. Resolution and super-resolution. Microsc. Res. Tech. 80, 590-598 (2017).

504 5. Power, R. M. \& Huisken, J. Adaptable, illumination patterning light sheet microscopy. Sci. Rep. 8, $505 \quad 1-11(2018)$

506 6. Štefko, M., Ottino, B., Douglass, K. M. \& Manley, S. Autonomous illumination control for $507 \quad$ localization microscopy. Opt. Express 26, 30882-30900 (2018).

508 7. Heel, M. Van. Similarity measures between images. Ultramicroscopy 21, 95-100 (1987).

509 8. Saxton, W. \& Baumeister, W. The correlation averaging of a regularly arranged bacterial cell $510 \quad$ envelope protein. J. Microsc. 127, 127--138 (1982).

511 9. Harauz, G. \& van Heel, M. Exact filters for general geometry three dimensional reconstruction. $512 \quad$ Optik 78, 146-156 (1986).

513 10. Rosenthal, P. B. \& Henderson, R. Optimal determination of particle orientation, absolute hand, 514 and contrast loss in single-particle electron cryomicroscopy. J. Mol. Biol. 333, 721-745 (2003).

515 11. Orlova, E. V. et al. Structure of keyhole limpet hemocyanin type $1(\mathrm{KLH} 1)$ at $15 \AA$ resolution by 516 electron cryomicroscopy and angular reconstitution. J. Mol. Biol. 271, 417-437 (1997). 
517 12. Unser, M., Trus, B. L. \& Steven, A. C. A new resolution criterion based on spectral signal-to-noise 518 ratio. Ultramicroscopy 23, 39-52 (1987).

519 13. Banterle, N., Bui, K. H., Lemke, E. A. \& Beck, M. Fourier ring correlation as a resolution criterion 520 for super-resolution microscopy. J. Struct. Biol. 183, 363-367 (2013).

521 14. Nieuwenhuizen, R. P. J. et al. Measuring image resolution in optical nanoscopy. Nat. Methods $522 \quad 10,557-562(2013)$.

523 15. Tortarolo, G., Castello, M., Diaspro, A., Koho, S. \& Vicidomini, G. Evaluating image resolution in 524 stimulated emission depletion microscopy. Optica 5, 32 (2018).

525 16. Raab, M. et al. Using DNA origami nanorulers as traceable distance measurement standards and 526 nanoscopic benchmark structures. Sci. Rep. 8, 1780 (2018).

527 17. Abràmoff, M. D., Magalhães, P. J. \& Ram, S. J. Image processing with ImageJ. Biophotonics Int. $528 \quad 11,36-42(2004)$.

529 18. Minsky, M. Memoir on inventing the confocal scanning microscope. Scanning 10, 128-138 $530 \quad$ (1988).

531 19. Hell, S. W. \& Wichmann, J. Breaking the diffraction resolution limit by stimulated emission: 532 stimulated-emission-depletion fluorescence microscopy. Opt. Lett. 19, 780 (1994).

533 20. Vicidomini, G., Bianchini, P. \& Diaspro, A. STED super-resolved microscopy. Nat. Methods 15, $534 \quad 173-182(2018)$.

535 21. Tortarolo, G., Sun, Y., Teng, W., Ishitsuka, Y. \& Vicidomini, G. Photon-separation to enhance the $536 \quad$ spatial resolution of pulsed STED microscopy. Nanoscale 11, 1754-1761 (2019).

537 22. Westphal, V. \& Hell, S. W. Nanoscale resolution in the focal plane of an optical microscope. 
Phys. Rev. Lett. 94, 1-4 (2005).

539

540

541

542

543

544

545

546

547

548

549

550

551

552

553

554

555

556

557

558

23. Oracz, J., Westphal, V., Radzewicz, C., Sahl, S. J. \& Hell, S. W. Photobleaching in STED nanoscopy and its dependence on the photon flux applied for reversible silencing of the fluorophore. Sci. Rep. 7, 11354 (2017).

24. Vicidomini, G., Moneron, G., Eggeling, C., Rittweger, E. \& Hell, S. W. STED with wavelengths closer to the emission maximum. Opt. Express 20, 5225-5236 (2012).

25. Galiani, S. et al. Strategies to maximize the performance of a STED microscope. Opt. Express 20, 7362-7374 (2012).

26. Heintzmann, R. \& Cremer, C. G. Laterally modulated excitation microscopy: improvement of resolution by using a diffraction grating. (International Society for Optics and Photonics, 1999).

27. Frohn, J. T. Super-resolution fluorescence microscopy by structured ligth illumination. (ETH Zürich, 2000).

28. Gustafsson, M. G. L. Surpassing the lateral resolution limit by a factor of two using structured illumination microscopy. J. Microsc. 198, 82-87 (2000).

29. Heintzmann, R. \& Huser, T. Super-Resolution Structured Illumination Microscopy. Chem. Rev. 117, 13890-13908 (2017).

30. Demmerle, J. et al. Strategic and practical guidelines for successful structured illumination microscopy. Nat. Protoc. 12, 988-1010 (2017).

31. Müller, M., Mönkemöller, V., Hennig, S., Hübner, W. \& Huser, T. Open-source image reconstruction of super-resolution structured illumination microscopy data in ImageJ. Nat. Commun. 7, 1-6 (2016). 
559 32. Dertinger, T., Colyer, R., lyer, G., Weiss, S. \& Enderlein, J. Fast, background-free, 3D super-

560 resolution optical fluctuation imaging (SOFI). Proc. Natl. Acad. Sci. U. S. A. 106, 22287-92 (2009).

561 33. Dertinger, T., Colyer, R., Vogel, R., Enderlein, J. \& Weiss, S. Achieving increased resolution and 562 more pixels with Super-resolution Optical Fluctuation Imaging (SOFI). Opt. Express 18, 18875$56318885(2010)$

564 34. Deschout, H. et al. Complementarity of PALM and SOFI for super-resolution live-cell imaging of 565 focal adhesions. Nat. Commun. 7, 13693 (2016).

566 35. Geissbuehler, S. et al. Mapping molecular statistics with balanced super-resolution optical 567 fluctuation imaging (bSOFI). Opt. Nanoscopy 1, 4 (2012).

36. Peeters, Y. et al. Correcting for photodestruction in super-resolution optical fluctuation imaging. 569 Sci. Rep. 7, 10470 (2017).

570

37. Rust, M. J., Bates, M. \& Zhuang, X. W. Sub-diffraction-limit imaging by stochastic optical

38. Betzig, E. et al. Imaging intracellular fluorescent proteins at nanometer resolution. Science 313, $1642-5$ (2006).

574

575

39. Sauer, M. \& Heilemann, M. Single-Molecule Localization Microscopy in Eukaryotes. Chem. Rev. 117, 7478-7509 (2017).

40. Marsh, R. J. et al. Artifact-free high-density localization microscopy analysis. Nat. Methods 15, 689 (2018).

578 41. Legant, W. R. et al. High-density three-dimensional localization microscopy across large 579 volumes. Nat. Methods 13, 359-365 (2016). 
580

581

582

583

584

585

586

587

588

589

590

591

592

593

594

595

596

597

598

599

600

601

42. Ovesny, M., Krizek, P., Borkovec, J., Svindrych, Z. \& Hagen, G. M. ThunderSTORM : a comprehensive ImageJ plug-in for PALM and STORM data analysis and super-resolution imaging. Bioinformatics 30, 2389-2390 (2014).

43. Fölling, J. et al. Photochromic Rhodamines Provide Nanoscopy with Optical Sectioning **. Angew. Chemie - Int. Ed. 46, 6266-6270 (2007).

44. Bossi, M. et al. Multicolor Far-Field Fluorescence Nanoscopy through Isolated Detection of Distinct Molecular Species. Nano Lett. 8, 2463-2468 (2008).

45. Lambert, T. J. \& Waters, J. C. Navigating challenges in the application of superresolution microscopy. J. Cell Biol. 216, 53-63 (2016).

46. Mikhaylova, M. et al. Resolving bundled microtubules using anti-tubulin nanobodies. Nat. Commun. 6, 1-7 (2015).

47. Pleiner, T., Bates, M. \& Görlich, D. A toolbox of anti - mouse and anti - rabbit IgG secondary nanobodies. J. Cell Biol. 217, 1143-1154 (2017).

48. Schmied, J. J. et al. Fluorescence and super-resolution standards based on DNA origami Flaws in evaluation schemes for pair- input computational predictions. Nat. Methods 9, 1133-1134 (2012).

49. Annibale, P., Vanni, S., Scarselli, M., Rothlisberger, U. \& Radenovic, A. Quantitative Photo Activated Localization Microscopy : Unraveling the Effects of Photoblinking. PLoS One 6, e22678 (2011).

50. Chazeau, A., Katrukha, E. A., Hoogenraad, C. C. \& Kapitein, L. C. Studying neuronal microtubule organization and microtubule-associated proteins using single molecule localization microscopy. Methods Cell Biol. 131, 127-149 (2016). 
